Article type : Full length original research paper

\title{
A universal automated tool for reliable detection of seizures in rodent models of acquired and genetic epilepsy
}

Authors: Pablo M. Casillas-Espinosa ${ }^{1,2 *}$, Armen $\operatorname{Sargsyan}^{3 *}$, Dmitri Melkonian ${ }^{3}$, Terence J. O'Brien

\section{Affiliations:}

${ }^{1}$ Departments of Neuroscience and Medicine, Central Clinical School, Monash University, VIC, 3004, Australia

${ }^{2}$ Department of Medicine, The Royal Melbourne Hospital, The University of Melbourne, VIC, 3052, Australia

${ }^{3}$ Kaoskey Pty. Ltd. Sydney, Australia

${ }^{4}$ Department of Neurology, The Alfred Hospital, Commercial Road, Melbourne, Victoria, 3004 Victoria, Australia.

${ }^{5}$ Department of Neurology, The Royal Melbourne Hospital, Grattan Street, Parkville, Victoria, 3050 Victoria, Australia.

*Authors contributed equally to this work

To whom correspondence should be addressed:

Name: Professor Terence J. O’Brien

Address: Department of Neuroscience, Central Clinical School, Monash University, 99 Commercial Road, Melbourne, 3004, Victoria, Australia

Phone: +61 399030855

E-mail address: terence.obrien@monash.edu

Keywords: automated seizure detection, TBI, post-SE, GAERS, posttraumatic epilepsy, WAG/Rij

This is the author manuscript accepted for publication and has undergone full peer review but has not been through the copyediting, typesetting, pagination and proofreading process, which may lead to differences between this version and the Version of Record. Please cite this article as doi: 10.1111/EPI.14691

This article is protected by copyright. All rights reserved 
Number of text pages - 26

Number of words (summary) - 298

Number of words (main text) -3998

Number of tables (main) - 2

Number of figures (main) -4 ( 2 in color)

Number of tables (supplementary) - 0

Number of figures (supplementary) - 5 (4 in color)

Supplementary information has been provided by Supplementary material (1-3) and supplementary figures (S1-S5).

\section{Summary}

Objective: Prolonged EEG monitoring in chronic epilepsy rodent models has become an important tool in pre-clinical drug development of new therapies, in particular those for anti-epileptogenesis, disease modification and drug resistant epilepsy. We have developed an easy-to-use, reliable, computational tool for automated detection of electrographic seizures from prolonged EEG recordings in rodent models of epilepsy. Methods: We applied a novel method based on advanced time-frequency analysis that detects the EEG episodes with excessive activity in certain frequency bands. The method uses an innovative technique of short-term spectral analysis -the Similar Basis Function algorithm. The method was applied for off-line seizure detection from longterm EEG recordings from four spontaneously-seizing, chronic epilepsy rat models: the fluid percussion injury ( $n=5$ rats, $n=49$ seizures) and post-status epilepticus model ( $n=119$ rats, $n=993$ seizures) of acquired epilepsy; and two genetic models of absence epilepsy - GAERS and WAG/Rij ( $\mathrm{n}=41$ and 14 rats; $n=8733$ and 825 seizures respectively)

Results: Our comparative analysis revealed that the EEG amplitude spectra of these four rat models is remarkably similar during epileptiform activity and have a single expressed peak within 17-25 Hz frequency range. Focusing on this band, our computer program detected all of seizures in the 179 rats. A quick semi-automated user inspection of the EEGs for the period of each identified event allowed quick 
rejection of artefact events. The overall processing time for 12 day-long recordings varied from few minutes (5-10) to 30min, depending on the number of artefact events, which was strongly correlated with the signal quality of the raw EEG data.

Significance: Our automated seizure detection tool provides high sensitivity, with acceptable specificity, for long and short-term EEG recordings from both acquired and genetic chronic epilepsy rat models. This tool has the potential to improve the efficiency and rigor of pre-clinical research and therapy development using these

models.

Keywords: automated seizure detection, TBI, post-SE, GAERS, posttraumatic epilepsy

\section{Key Points}

- The "Assyst" novel and versatile algorithm detected $100 \%$ of the 10,600 seizures, which represents over 76000 hours of EEG recordings.

- We discovered a frequency band of 17 to $25 \mathrm{~Hz}$ that is specific to detect all of the seizures in the post-SE, post-TBI, GAERS and WAG/Rij rodent models of acquired and genetic epilepsy.

- Processing and reviewing 24h of EEG recordings took an average of 1 minute per $24 \mathrm{~h}$ of EEG recordings, which represents a saving $90-98 \%$ of time to review pre-clinical EEG.

\section{Introduction}

Epilepsy affects 50 million people worldwide (World Health Organization, 2006), and poses a significant burden on the quality of life of affected individuals and their families. Since the discovery of bromide as the first anti-seizure drug, there has been an impressive expansion of clinically effective therapies that decrease the frequency and severity of seizures in people with epilepsy. The newer anti-seizure drugs have 
been identified through systematic screening in batteries of an increasing number of in vivo and in vitro seizure and epilepsy models ${ }^{1}$.

However, the focus of translational research and drug development for the epilepsies has now shifted from the development of more antiseizure drugs, to the development of anti-epileptogenic and disease modifying therapies. Prolonged EEG recordings in chronic epilepsy models are a critical part of the drug development testing paradigm for these therapies ${ }^{2 ; 3},{ }^{4-6}$. For this an efficient and accurate measure of quantitating the occurrence of spontaneous seizures is critical ${ }^{3}$, .

For the study of acquired epilepsy, the two most common used models are the poststatus epilepticus model (post-SE) ${ }^{6}$, and the traumatic brain injury induced posttraumatic epilepsy (PTE) models ${ }^{8}$. On the other hand, the Genetic Absence Epilepsy rats from Strasbourg (GAERS) and Wistar Albino Glaxo/from Rijswijk (WAG/Rij) are the most widely used models to study genetic generalized epilepsy with absence seizures $4 ; 5$. Prolonged EEG monitoring is necessary to phenotype the occurrence of spontaneous seizures in different animal models and to evaluate the acute and chronic effects of the novel interventions ${ }^{9}$. Studies utilizing EEG typically involve comparison of measurements obtained from different experimental groups, or from the same experimental group at different times. Given the heterogeneity of epilepsy and, in some cases, the low frequency of spontaneous occurring seizures, to appropriately power anti-epileptogenesis and disease modification studies, is mandatory to have large number of animals per cohort as well as multiple and prolonged periods of recording to establish if the experimental treatments are successful in preventing or modifying the progression of epilepsy $2 ; 3 ; 7 ; 9$.

Therefore, it is critical to quantify the presence and number of seizures in pre-clinical studies. Although electroencephalographic seizures (EEG seizures) can be relatively easy to recognize during expert visual inspection of the EEG, the overall process to analyze EEG recordings is very time consuming. Moreover, it requires a significant amount of time to train a new EEG reviewer, and the manual analysis is prone to errors mostly related to fatigue and eyestrain. Thus, an automatic seizure detection method would greatly increase the throughput and would help to standardize and increase the reproducibility of the analysis in large-scale pre-clinical trials. 
Here we report a user-friendly, easy to use, reliable, computational tool for detection of EEG seizures from prolonged EEG recordings in rodent models of epilepsy, and validate this in prolonged EEG recordings from four rat models of chronic epilepsy two acquired and two genetic. The tool is based on a novel signature in the 17 to 25 $\mathrm{Hz}$ frequency band of the EEG that is specific to detect all of the EEG seizures in these four rodent models.

\section{Methods}

\section{Animals}

11-week-old male Wistar rats were used for the Post-SE and PTE models of acquired epilepsy (at the time of application of the epileptogenic insult). 24-week-old male GAERS and WAG/Rij rats were used in the experiments. All procedures were approved by the Florey Animal Ethics Committee (ethics number 14-072 UM). The animals where individually housed with alternating 12 hour light and dark cycles. Food and water were provided ad libitum for the whole duration of the study.

\section{Kainic acid induced post-status epilepticus of acquired epilepsy}

A repeated low dose kainic acid (KA) administration protocol was used as previously described ${ }^{10}$. Rats were injected i.p. with an initial dose of KA $7.5 \mathrm{mg} / \mathrm{kg}$. Animals

were monitored for behavioral seizures based on the Racine scale ${ }^{11 ; 12}$. If no selfsustained seizure activity was observed with at least five class IV-V seizure of Racine, another i.p. dose of $2.5 \mathrm{mg} / \mathrm{kg}$ of $\mathrm{KA}$ was administered up to a maximum of $15 \mathrm{mg} / \mathrm{kg}$. An animal was eliminated from the experiment if it didn't show a self-sustained SE after a maximum KA dose. SE was stopped after 4 hours with diazepam $(5 \mathrm{mg} / \mathrm{kg} / \mathrm{dose})$. After recovery the animals were returned to their home cages in the animal house and kept in routine housing conditions until they were implanted with EEG electrodes for the prolonged EEG recordings (as described below). 


\section{Induction of post-traumatic epilepsy}

Wistar rats received a lateral fluid percussion injury (FPI) as previously described ${ }^{13}$; ${ }^{14}$. Briefly, with the animal under anesthesia, a 5-mm craniotomy positioned 4-mm right lateral and 4-mm posterior to Bregma, was performed to create a circular window exposing the intact dura mater of the brain. A modified female Luer-Lock cap was secured over the craniotomy window by dental acrylic. A severe intensity (320-350 kPa) fluid pulse of silicone oil generated by the fluid percussion device was delivered to the brain. On resumption of spontaneous breathing, and return to pre- FPI levels of heart rate and oxygenation status, the dental acrylic caps were removed and the wound sutured closed. This injury results in PTE in $30-50 \%$ of rats at 6 months ${ }^{13-}$ ${ }^{17}$. After recovery the animals were returned to their home cages in the animal house and kept in routine housing conditions until they were implanted with EEG electrodes for the prolonged EEG recordings (as described below).

\section{EEG electrode implantation surgery}

EEG Surgery was performed 6 weeks after SE or FPI, or at 6 months of age for the GAERS and WAG/Rij, as previously described ${ }^{18}$. Briefly, animals were anesthetized with isoflurane. Six burr holes were drilled through the skull without penetrating the dura, one on each side anterior to the Bregma, two to each side anterior to lambda and two to each side in the parietal bones. Ground and reference were placed in the occipital bone. Stainless steel subdural screw recording electrodes (Plastics One, Australia) were placed screwed into each hole. The recording electrodes were fixed in position by applying Vertex dental cement around the electrodes and over the skull.

\section{EEG recordings}

EEG were acquired using Profusion 5 software (Compumedics, Australia) unfiltered and digitized at $512 \mathrm{~Hz}$ using a tethered EEG cable system with the rats freely moving 
in their home cages. EEG recordings were acquired for 2-4 weeks continuously in the post-SE and FPI rats whilst GAERS and WAG/Rij had 48h of continuous EEG recordings. Only animals that presented with EEG seizures on the EEG were selected for the study. A referential montage was used.

\section{Manual seizure analysis}

EEG analysis was performed in a blinded manner and confirmed by two different expert observers. All EEG recordings were visually and manually annotated using Profusion 5 software. For the post-SE and PTE animals, an electroencephalographic seizure was defined as an episode of rhythmic spiking activity that was three times the baseline amplitude and a frequency $>5 \mathrm{~Hz}$ that last at least $10 \mathrm{~s}$. The end of a seizure was determined as the last spike. ${ }^{17 ; 19 ; 20}$.

For GAERS and WAG/Rij, an electroencephalographic seizure was defined as spike and wave discharge (SWD) of amplitude of more than three times baseline, a frequency of 7-12 Hz, and duration of longer than $0.5 \mathrm{~s}^{4 ; 5 ; 21 ; 22}$. The start and end of each seizure was determined by manually marking the beginning and end of each SWD on the EEG. For all of the animals the total number of seizures was quantified.

\section{Automated seizure analysis}

We developed a novel software tool, "Assyst", written in Delphi (dialect of Object Pascal, Embarcadero Technologies, Austin, TX, USA) that is aimed to significantly facilitate the detection of electrographic seizures in prolonged EEG recordings in rodent models of epilepsy. The seizure detection method implemented in the "Assyst" and presented here utilizes an advanced time-frequency analysis of the signal from a single or multiple EEG channels to reveal the EEG segments with excessive activity in a certain frequency band. We assessed the spectral content of the EEG within a given frequency band using a measure we refer to as Spectral band index (SBI), which we calculate over time using a pre-determined running time window. 
The main steps of the algorithm are presented in Figure 1. The algorithm consists of main (shaded block) and optional (white blocks) steps. The proposed algorithm is interactive; some operations are automatically performed by the computer, others require user input.

\section{Input data}

EEG recordings are exported unfiltered into European data format (.edf) files and then are loaded to the "Assyst" software. There are no restrictions on the duration of the EEG recordings, data size or number of channels to be analyzed.

\section{Time-Frequency analysis - calculation of spectral band index}

This step is aimed to reveal the temporal dynamics of a particular frequency component of the EEG. To achieve this, we define the frequency range of interest, (frequency band), define the length of EEG segments (time window) that will be used to calculate the power spectrum of the EEG, and move this window along the EEG with certain time step. For each window position (Figure 2), the power spectrum of the EEG segment is calculated within the defined frequency range using a modification of our Similar Basis Function algorithm for Fourier Transform ${ }^{23}$. The maximum value of the power spectrum in this frequency range is found, and this parameter - the SBI - is used as an estimate for spectral intensity of the EEG within this frequency band. The SBI values are then plotted against time at centers of consecutive windows to form the SBI curve (Figure 2).

\section{Event selector}

To find the times of high electrical EEG activity within the frequency band of interest, suggestive of seizures we define a threshold value of the SBI. This threshold allows to filter background EEG signal and interictal activity, from the seizures. The automatic threshold definition is based on building a distribution histogram of SBI values over entire recording time (Supplementary material 1). Assuming that the total duration of ictal events is only a small fraction of overall duration of the recording, the region of the histogram with highest density of distribution will indicate the interictal SBI value range. By setting the threshold above this range, we will cut off the "ordinary" values of the SBI and leave only the seizure events (Supplementary Figure S1). 
After the threshold is defined, all episodes of the EEG for which the SBI is above the threshold are separated in a list of events that also specifies their start and end times. If multiple channels are analyzed, the algorithm groups the overlapping events from different channels, so they can be counted as one event. The start and end times of the selected events are roughly determined as the points where the SBI curve crosses the threshold (Supplementary Figure S1). The precision of defining the starts and ends in this way is equal to the size of time window. However, the software allows user to redefine the start and end times of selected events manually, simply moving special cursors to desired positions on the EEG. The Assyst software is able to show the signal of single or multiple channels to allow the user for a more efficient review of the events.

\section{Artefact removal and user inspection of selected events}

An optional automatic artefact removal procedure in our method identifies and eliminates false positives (FP) that were caused by strong artefacts. It is performed after the threshold-based event selection. The procedure examines the selected events for presence of sharp artefacts by analyzing the signal's derivative and finding the pieces of EEG with sharp and large deflections in amplitude (Supplementary material $3)$.

The final stage of processing requires the input from the user. At this stage the user visually examines the selected events by reviewing the EEG either to confirm them as electrographic seizures or reject them as false detections.

\section{Comparison of Assyst and manual seizure detection and algorithm performance assessment}

All of the EEG recordings were manually analyzed as described previously by two blinded experts. Assyst software was used to analyze the same EEG recordings and the results obtained by the Assyst were confirmed by two independent blinded reviewers. The total number of the EEG seizures detected on each method, and the time to review each file were analyzed. The main criterion for the algorithm performance assessment was the percentage of real seizures detected by the algorithm during the automatic event detection (sensitivity). 
The second criterion was the comparison of total time spent for the analysis of one day (24 hours) of recording using the Assyst software.

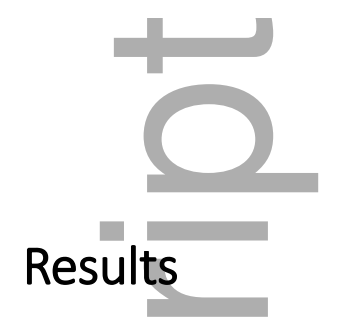

\section{Automated seizure detection}

The method was applied for off-line EEG seizure detection from long-term EEG recordings from post-SE, PTE, GAERS and WAG/Rij. We processed recordings from 179 rats that were representatives of four different rat models of epilepsy. The EEG recordings contained in total 10,600 seizures, which represents over 76000 hours of EEG recordings, Table 1 . The average duration of records from Post-SE and PTE animals was 24.6 days. The records from GAERS and WAG/Rij animals were shorter 24.2 hours $(+/-3.4)$.

For the Post-SE (n=119 rats, n=993 seizures, 2.8 seizures per week) and PTE (n=5 rats, $n=49$ seizures, 28 seizures per week) the EEG seizure detection was performed using the following sets of parameters. The running window size was 10s, the window step was $5 \mathrm{~s}$, and the frequency band was from 20 to $23 \mathrm{~Hz}$.

For GAERS ( $n=41, n=8733$ seizures) and WAG/Rij ( $n=14$ rats, $n=825$ seizures) the parameters for automated seizure detection were slightly different. We used shorter windows, $2-5$ seconds for GAERS and 5 seconds for WAG/Rij to reliably detect the shorter SWD of these genetically inbred species. The window step was equal to the half of window size. Also, the EEG seizures in both the GAERS and WAG/Rij showed slightly higher variability in the frequency of the component we were focusing on (see Supplementary Figure S2), and thus a wider frequency band of 17 to $25 \mathrm{~Hz}$ was used in the SBI calculation.

Examples of electrographic seizures in Post-SE, PTE, GAERS and WAG/Rij models are shown in Figure 3. Note that the average discharge frequency (the repetition rate of spikes) significantly differs between the models: $6 \mathrm{~Hz}$ for Post-SE, $2.4 \mathrm{~Hz}$ for PTE, 
7.3 Hz for GAERS and $9 \mathrm{~Hz}$ for WAG/Rij. Meanwhile, the waveforms of individual spike-waves from different rat models (Figure $3 \mathrm{~B}$ ) have similar structure and temporal dynamics. Due to this temporal similarity, they have also similar amplitude frequency characteristics, with a single peak in the range 17-25 Hz (Supplementary Figure S2).

Figure 4 illustrates the specificity of the $17-25 \mathrm{~Hz}$ frequency component to electroencephalographic seizures. This narrow band shows a significant difference between SBI values corresponding to ictal events and those corresponding to background interictal activity. It is important to note that the smallest ictal SBI value is more than 10 times larger than the largest inter-ictal SBI value, which provides a very safe margin for threshold to detect seizures.

In Supplementary material 2 we compare and discuss the SBI curves calculated for the same recording for wide and narrow frequency bands (Supplementary Figure S3).

\section{Artefact removal}

The majority of analyzed records contained artefacts. The optional procedure for automatic identification and removal of detections caused by artefacts was performed after the initial threshold-based event selection. A conservative removal algorithm was applied to avoid erroneous removal of ictal events (Supplementary material 3 and Supplementary Figures S4-S5).

It is important to note that the number of true seizures detected with our algorithm does not depend on the use of the artefact removal tool. Rather, the artefact removal reduces the FP rate and the time it takes to manually review the selected events. In all of the Post-SE and PTE recordings the automatic artefact removal procedure removed on average $38.5 \%$ of the artefacts that were initially selected by the algorithm.

In Post-SE and PTE EEG recordings that did not contain artefacts $(n=7)$ or contained a few (Figure 4 and Supplementary Figure S3) the number of FPs was zero. In these cases, the specificity of the algorithm with or without the use of the artefact removal procedure was $100 \%$.

The EEG recordings from GAERS and WAG/Rij rats contained only a minimal number of artefacts, and the artefact removal procedure was not used. 


\section{Algorithm Performance Assessment}

We assessed the algorithm performance using two criteria: sensitivity (the percentage of real seizures detected by the algorithm) and time saving compared to manual seizure screening.

For all analyzed records, the comparison of the EEG seizures detected with the use of Assyst software with the previous manual scores made by experts did not reveal any missed seizure ( 0 false-negatives) i.e., the program detected $100 \%$ of EEG seizures in all records. This was verified afterwards by two independent experts (Table 1). Moreover, it was revealed that 4 seizures in the Post-SE animals and 6 seizures in PTE animals were not annotated in the manual analysis. The false positive rate was not affected by the duration of the EEG recordings.

Assyst significantly reduced the time to screen for seizures in EEG recordings. The longest time spent by processing a single channel EEG recording of one animal was about 5 minutes per 1 day of record, with an average of 1 minute per $24 \mathrm{~h}$ of EEG recordings (Table 2). For Post-SE and PTE recordings that contained minimal artefacts, the processing took about 6-10 seconds per day of recording. As expected, longer inspection time was required for GAERS and WAG/Rij records as each record contained hundreds of seizures per $24 \mathrm{~h}$ of EEG recordings.

In contrast, an experienced investigator spends 40-90 minutes for manual seizure screening through 1 day of EEG recordings. Comparing our average Assyst processing time with the manual seizure screening, the time needed to screen for seizures in any given set of EEG recordings reduced 60 times, which represents saving 90-98\% of time of the reviewer when using the Assyst.

\section{Discussion}


We present an effective and efficient automated algorithm which has a high sensitivity, detecting all of the seizures in different chronic animal models of genetic and acquired epilepsy in this study, and high specificity with only a small, acceptable, number of false positive seizure identifications. The Assyst method was applied for off-line seizure detection from long-term EEG recordings from four spontaneously seizing, chronic epileptic rat models: post-SE model of acquired epilepsy and the FPI model of PTE; and two genetic models of absence epilepsy - GAERS and WAG/Rij. Seizure detection algorithms have been described in the literature ${ }^{24 ; 25}$, most of which are aimed at human EEG analysis and very few are designed particularly for rodents ${ }^{26-35}$. However, no method has been described to detect all of the seizures in different animal models.

The method presented here belongs to the class of univariate time-frequency analysisbased methods ${ }^{23}$. The novelty is in the definition and in the way of calculation of the discriminative feature used - the Spectral band index (SBI), which is calculated for a narrow frequency band and is used to assess the spectral content of the EEG to distinguish the EEG seizures from inter-ictal activity.

We found that the ictal EEG in the examined rat models contains a strong component in the frequency range from 17 to $25 \mathrm{~Hz}$. This novel finding was critical to determine the choice for the discriminative feature of EEG seizures and ensured an effective performance of the algorithm. We found that this peculiarity comes from the frequency composition of single discharges, or individual spike and wave complexes within the seizures in these animals (Supplementary material 2, figure S2). Importantly, this frequency band component is not present anywhere else in the interictal EEG. This is reflected in that the power spectrum of the EEG (and the SBI) has much stronger values in this frequency range during the seizures than at any time between seizures (Figure 4).

Another key factor for the algorithm's performance was the accuracy of calculation of the power spectrum achieved by using our previously described Similar Basis Function (SBF) algorithm for numerical calculations of Fourier transforms ${ }^{23}$. Numerical estimation of Fourier transforms is usually based on procedures employing various algorithms of the fast Fourier transform (FFT) ${ }^{36}$. The latter is supported by a Fourier series model of the data, i.e. the addressees are periodic signals. This distinction with the Fourier integrals is a troublesome problem when functions of short duration, like EEG fragments extracted by employed windows, are transformed 
from the time to frequency domain. The major concern is the spectral leakage which may cause significant distortions of the frequency domain characteristics. The remedies of windowing and zero-padding usually introduce problems of their own. By contrast to the FFT, the SBF algorithm is an original version of Filon-type methods that provide maximum precision in the estimation of trigonometric integrals using interpolation polynomials of different degrees. Removal of spectral leakage provides means to calculate the power spectrum with high resolution and remarkable accuracy. In this study, we used a modified SBF algorithm optimized for calculation of uniformly sampled input signal, which is the case for EEG recordings. This optimization made the algorithm significantly faster and comparable in speed with the FFT.

Current implementation of the algorithm is not devoted to real time EEG processing because the threshold is defined on the basis of the entire record. Implementation of some off-line procedures was necessary to get the most accurate (for each particular recording) statistical estimate for the baseline SBI values. Using these results we are currently working on the on-line version of the algorithm, where the threshold will be defined dynamically based on the past values of SBI.

The majority of our records contained artefacts, both of physiological and nonphysiological (instrumental) nature. Most physiological artefacts did not have strong components in the analyzed frequency band, and thus were rarely detected by the algorithm, with the exception of strong ECG or chewing artefacts (Supplementary Figure S4 A, B). In contrast, the non-physiological artefacts were sometimes detected as events of interest because they mostly contain sharp and large amplitude signal that has strong spectral power within wide frequency diapason including the 17 to $25 \mathrm{~Hz}$ band (Supplementary Figure S4 C-F).

A variety of artefact detection and removal methods of different complexity is described in literature, though no single existing artefact detection method is universal. Importantly, but there is no method designed specifically for single channel recordings in rodents $37 ; 38$.

When artefact identification is applied in a seizure detection algorithm, there is a high risk of erroneously identifying a seizure as an artefact, especially if the seizure is indeed contaminated by artefacts, as it occurs normally in chronic EEG recordings we observed several times in our recordings ${ }^{38}$. Therefore, we decided to use a reliable and conservative procedure for artefact identification. This procedure did not 
erroneously remove any seizure in the studied records, and further reduced the user's inspection time on average by about $40 \%$.

False negatives are the most critical component for a reliable automated seizure detection method. One missed seizure during the automated detection would require a manual inspection for the entirety of the EEG recording file.

In our case, the sensitivity was $100 \%$ (i.e., zero false negatives) in all records from all the four epilepsy rat models. Specificity, which is determined by the number of false positives, significantly depended on the quality of recording. In recordings with a reduced number of artefacts, the number of FPs was zero, giving specificity of $100 \%$. In records containing large number of artefacts even after application of artefact removal procedure the number of FPs remained for visual inspection was relatively high (ranging from 0 to 193 / day, on average 32/day, or 1.3/h), which is comparable to what has been describe in the literature $39 ; 40$

Overall, we have shown a reliable automated tool that provides high sensitivity, detecting $100 \%$ of the EEG seizures in different animal models of genetic and acquired epilepsy. Our Assyst is versatile and a significant time-saving seizure detection program, which can facilitate high-throughput studies. Moreover, it provides remarkable flexibility in processing depending on particular data and user needs, from automatic with minimum influence from user to a semi-automated inspection of every peak, large or small. Moreover, the software provides flexibility to advance users to search for epileptiform events with smaller duration and amplitude or when dealing with new or uncharacterized models. The Assyst algorithm has the potential to improve the efficiency and rigor of pre-clinical research and therapy development using these models.

\section{Acknowledgements}

We thank Prof. Gilles van Luijtelaar (Radboud University Nijmegen, The Netherlands) for kindly providing the $\mathrm{WAG} / \mathrm{Rij}$ rat data. 


\section{Disclosure of Conflicts of Interest}

PMCE, AS, DM and TJO declare no conflict of interest.

\section{Ethical statement}

We confirm that we have read the Journal's position on issues involved in ethical publication and affirm that this report is consistent with those guidelines.

\section{References}

1. Galanopoulou AS, Buckmaster PS, Staley KJ, et al. Identification of new epilepsy treatments: issues in preclinical methodology. Epilepsia 2012;53:571-582.

2. Barker Haliski M, Friedman D, French J, et al. Disease modification in epilepsy: from animal models to clinical applications. Drugs 2015;75:749-767.

3. Galanopoulou A, Mowrey W. Not all that glitters is gold: A guide to critical appraisal of animal drug trials in epilepsy. Epilepsia Open 2016;1:86-101.

4. Coenen AM, Drinkenburg WH, Inoue M, et al. Genetic models of absence epilepsy, with emphasis on the WAG/Rij strain of rats. Epilepsy Res 1992;12:75-86.

5. Marescaux C, Vergnes M, Depaulis A. Genetic absence epilepsy in rats from Strasbourg--a review. J Neural Transm Suppl 1992;35:37-69.

6. Morimoto K, Fahnestock M, Racine RJ. Kindling and status epilepticus models of epilepsy: rewiring the brain. Prog Neurobiol 2004;73:1-60.

7. Galanopoulou AS, Simonato M, French JA, et al. Joint AES/ILAE translational workshop to optimize preclinical epilepsy research. Epilepsia 2013;54 Suppl $4: 1-2$.

8. Liu S-J, Zheng P, Wright D, et al. Sodium selenate retards epileptogenesis in acquired epilepsy models reversing changes in protein phosphatase $2 \mathrm{~A}$ and hyperphosphorylated tau. Brain 2016;139:1919-1938.

9. Kadam S, D'Ambrosio R, Duveau V, et al. Methodological standards and interpretation of video-electroencephalography in adult control rodents. 
A TASK1-WG1 report of the AES/ILAE Translational Task Force of the ILAE. Epilepsia 2017;58 Suppl 4:10-27.

10. Bhandare A, Kapoor K, Powell K, et al. Inhibition of microglial activation with minocycline at the intrathecal level attenuates sympathoexcitatory and proarrhythmogenic changes in rats with chronic temporal lobe epilepsy. Neuroscience 2017;350:23-38.

11. Racine RJ. Modification of seizure activity by electrical stimulation. I. Afterdischarge threshold. Electroencephalogr Clin Neurophysiol 1972;32:269-279.

12. Racine RJ. Modification of seizure activity by electrical stimulation. II. Motor seizure. Electroencephalogr Clin Neurophysiol 1972;32:281-294.

13. Liu SJ, Zheng P, Wright DK, et al. Sodium selenate retards epileptogenesis in acquired epilepsy models reversing changes in protein phosphatase $2 \mathrm{~A}$ and hyperphosphorylated tau. Brain 2016;139:1919-1938.

14. Shultz SR, Wright DK, Zheng P, et al. Sodium selenate reduces hyperphosphorylated tau and improves outcomes after traumatic brain injury. Brain 2015;138:1297-1313.

15. Kharatishvili I, Nissinen JP, McIntosh TK, et al. A model of posttraumatic epilepsy induced by lateral fluid-percussion brain injury in rats. Neuroscience 2006; 140:685-697.

16. Shultz SR, Cardamone L, Liu YR, et al. Can structural or functional changes following traumatic brain injury in the rat predict epileptic outcome? Epilepsia 2013;54:1240-1250.

17. Brady R, Casillas Espinosa P, Agoston D, et al. Modelling traumatic brain injury and posttraumatic epilepsy in rodents. Neurobiol Dis 2018.

18. Casillas Espinosa P, Powell K, Zhu M, et al. Evaluating whole genome sequence data from the Genetic Absence Epilepsy Rat from Strasbourg and its related non-epileptic strain. PLoS One 2017;12:e0179924-e0179924.

19. Van Nieuwenhuyse B, Raedt R, Sprengers M, et al. The systemic kainic acid rat model of temporal lobe epilepsy: Long-term EEG monitoring. Brain Res 2015;1627:1-11.

20. Pitkänen A, Kharatishvili I, Narkilahti S, et al. Administration of diazepam during status epilepticus reduces development and severity of epilepsy in rat. Epilepsy Res 2005;63:27-42. 
21. Powell KL, Cain SM, Ng C, et al. A Cav3.2 T-type calcium channel point mutation has splice-variant-specific effects on function and segregates with seizure expression in a polygenic rat model of absence epilepsy. $J$ Neurosci 2009;29:371-380.

22. Drinkenburg WH, Coenen AM, Vossen JM, et al. Spike-wave discharges and sleep-wake states in rats with absence epilepsy. Epilepsy Res 1991;9:218-224.

23. Melkonian D. Similar basis function algorithm for numerical estimation of Fourier integrals. Numer Algor 2010;54:73-100.

24. Koren J, Herta J, Fürbass F, et al. Automated Long-Term EEG Review: Fast and Precise Analysis in Critical Care Patients. Front Neurol 2018;9:454-454.

25. Ulate Campos A, Coughlin F, Gaínza Lein M, et al. Automated seizure detection systems and their effectiveness for each type of seizure. Seizure 2016;40:88101.

26. Xanthopoulos P, Liu C-C, Zhang J, et al. A robust spike and wave algorithm for detecting seizures in a genetic absence seizure model. Conf Proc IEEE Eng Med Biol Soc 2009;2009:2184-2187.

27. van Luijtelaar G, Lüttjohann A, Makarov V, et al. Methods of automated absence seizure detection, interference by stimulation, and possibilities for prediction in genetic absence models. J Neurosci Methods 2016;260:144-158.

28. Ovchinnikov A, Lüttjohann A, Hramov A, et al. An algorithm for real-time detection of spike-wave discharges in rodents. J Neurosci Methods 2010;194:172-178.

29. Aghazadeh R, Shahabi P, Frounchi J, et al. An autonomous real-time singlechannel detection of absence seizures in WAG/Rij rats. Gen Physiol Biophys 2015;34:285-291.

30. Van Hese P, Martens JP, Boon P, et al. Detection of spike and wave discharges in the cortical EEG of genetic absence epilepsy rats from Strasbourg. Phys Med Biol 2003;48:1685-1700.

31. Richard CD, Tanenbaum A, Audit B, et al. SWDreader: a wavelet-based algorithm using spectral phase to characterize spike-wave morphological variation in genetic models of absence epilepsy. J Neurosci Methods 2015;242:127-140.

32. White A, Williams P, Hellier J, et al. EEG spike activity precedes epilepsy after kainate-induced status epilepticus. Epilepsia 2010;51:371-383. 
33. Niknazar M, Mousavi SR, Motaghi S, et al. A unified approach for detection of induced epileptic seizures in rats using ECoG signals. Epilepsy Behav 2013;27:355-364.

34. Lee J, Park J, Yang S, et al. Early Seizure Detection by Applying FrequencyBased Algorithm Derived from the Principal Component Analysis. Front Neuroinform 2017;11:52-52.

35. Dheer P, Chaitanya G, Pizarro D, et al. Seizure Detection and Network Dynamics of Generalized Convulsive Seizures: Towards Rational Designing of ClosedLoop Neuromodulation. Neurosci J 2017;2017:9606213-9606213.

36. Rao KR, Kim DN, Hwang JJ. Fast Fourier Transform: Algorithms And Applications. Springer: Dordrecht Heidelberg 2010.

37. Islam M, Rastegarnia A, Yang Z. Methods for artifact detection and removal from scalp EEG: A review. Neurophysiol Clin 2016;46:287-305.

38. Islam M, Rastegarnia A, Yang Z. A Wavelet-Based Artifact Reduction From Scalp EEG for Epileptic Seizure Detection. IEEE J Biomed Health Inform 2016;20:1321-1332.

39. White A, Williams P, Ferraro D, et al. Efficient unsupervised algorithms for the detection of seizures in continuous EEG recordings from rats after brain injury. J Neurosci Methods 2006;152:255-266.

40. Andrade P, Paananen T, Ciszek R, et al. Algorithm for automatic detection of spontaneous seizures in rats with post-traumatic epilepsy. J Neurosci Methods 2018;307:37-45.

\section{Figure legends}

Figure 1. Block-diagram of the algorithm. Shaded blocks: main steps; white blocks: optional steps. The blocks on the left side indicate operations performed by user, and blocks on the right side by the computer.

Figure 2. Diagram showing calculation of SBI curve. A) Fragment of EEG and the current position of running time window. B) The FFT and the power spectrum of EEG segment from this window is calculated using the SBF algorithm, for the frequency range $\left[f_{1}, f_{2}\right]$. The maximum value of the power spectrum in this range is 
the SBI of current window. C) The SBIs of consecutive time windows are then plotted against time and form the SBI curve.

Fig. 3. A) Examples of electrographic seizures (10 s fragments) from four rat models. B) $150 \mathrm{~ms}$ fragments of traces in A showing single spike waves (marked by asterisk in corresponding trace in A).

Figure 4. Narrow band seizure detection. Upper trace shows the SBI curve calculated for 12 days (288 hours)-long EEG recording from a Post-SE rat that had four seizures during the recording period. The bottom traces show zoomed fragments of the SBI curve corresponding to the first and second seizures that have the smallest and the largest SBI values, correspondingly, among all ictal events, and the inter-ictal event with largest SBI value. The SBI peak values are in relative units.

\section{Tables}

Table 1. Total number of seizures analyzed.

\begin{tabular}{|c|c|c|c|c|c|c|c|}
\hline \multirow{2}{*}{ Model } & Numb & \multicolumn{6}{|c|}{ Number of events } \\
\hline & $\begin{array}{c}\text { er of } \\
\text { animal }\end{array}$ & $\begin{array}{c}\text { Manuall } \\
y \\
\text { Annotat } \\
\text { ed by } \\
\text { expert }\end{array}$ & $\begin{array}{c}\text { Automatica } \\
\text { lly selected } \\
\text { by event } \\
\text { selector }\end{array}$ & $\begin{array}{c}\text { Remove } \\
\mathrm{d} \text { by } \\
\text { artefact } \\
\text { removal } \\
\text { procedu } \\
\text { re }\end{array}$ & $\begin{array}{c}\text { Reject } \\
\text { ed by } \\
\text { the } \\
\text { user }\end{array}$ & $\begin{array}{c}\text { Classifi } \\
\text { ed as } \\
\text { seizures } \\
\text { by the } \\
\text { user }\end{array}$ & $\begin{array}{c}\text { Verified } \\
\text { by two } \\
\text { independe } \\
\text { nt experts }\end{array}$ \\
\hline Post-SE & 119 & 989 & 32877 & 13204 & 18680 & 993 & 993 \\
\hline PTE & 5 & 43 & 1538 & 171 & 1318 & 49 & 49 \\
\hline GAERS & 41 & 8,733 & 9412 & $\begin{array}{l}\text { not } \\
\text { applied }\end{array}$ & 679 & 8,733 & 8,733 \\
\hline $\begin{array}{l}\text { WAG/ } \\
\text { Rij }\end{array}$ & 14 & 825 & 856 & $\begin{array}{l}\text { not } \\
\text { applied }\end{array}$ & 31 & 825 & 825 \\
\hline Total & 179 & 10,590 & 44683 & 13375 & 20708 & 10,600 & 10,600 \\
\hline
\end{tabular}


Table 2. Processing times of $24 \mathrm{~h}$ of EEG recordings

\begin{tabular}{|l|l|l|}
\hline & Processing time per 1 day of EEG recordings & Minutes \\
\hline Assyst & Max & 5 \\
\hline & Min & 0.1 \\
\hline & Average & 1 \\
\hline Manual & $\begin{array}{l}\text { Approximate time spent by expert for visual } \\
\text { examination of 1 day EEG recordings }\end{array}$ & $40-90$ \\
\hline & & \\
\hline
\end{tabular}
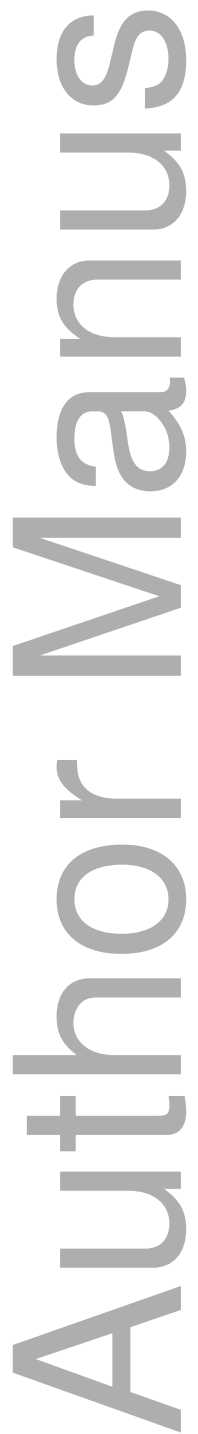


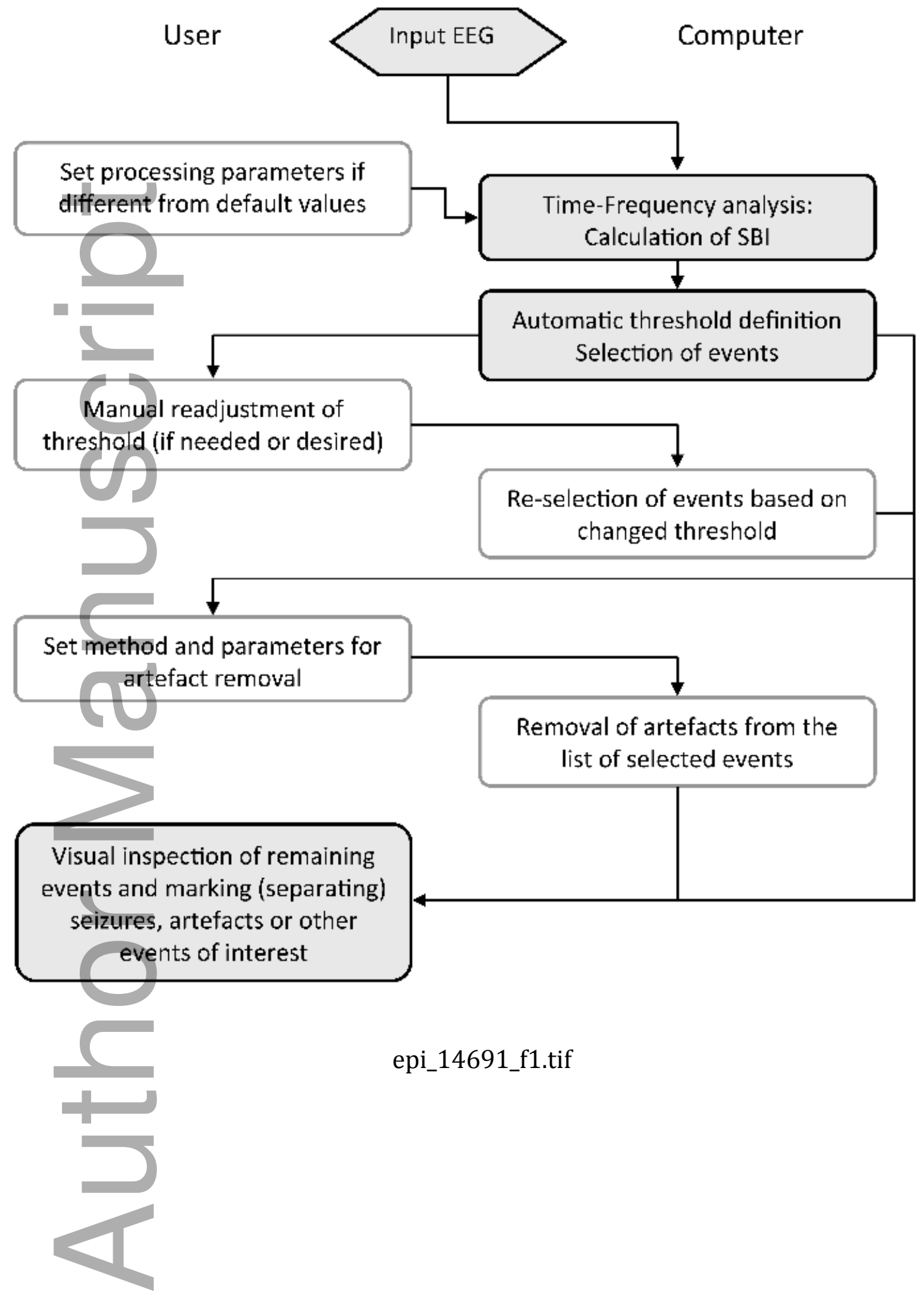

This article is protected by copyright. All rights reserved 

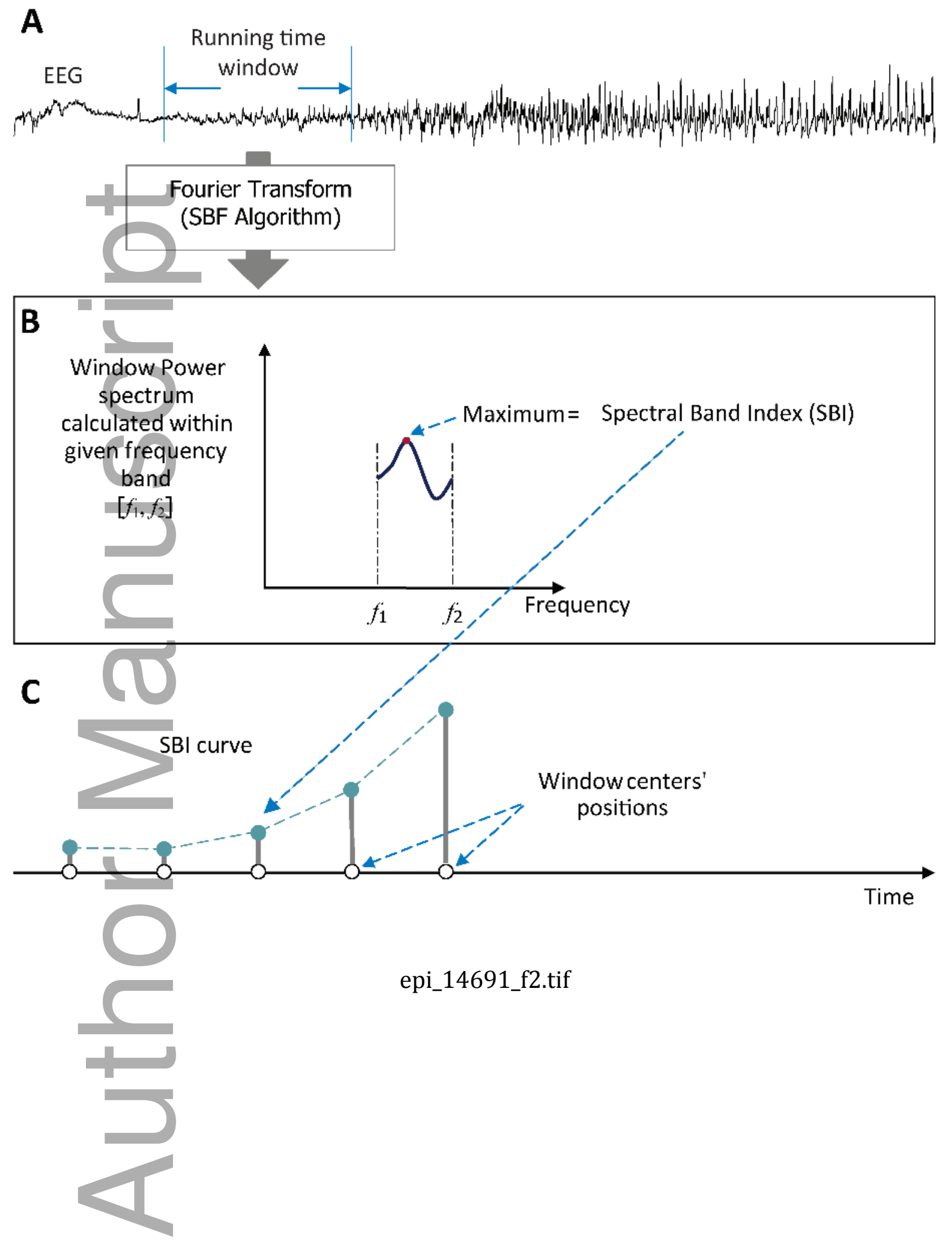

This article is protected by copyright. All rights reserved 
A
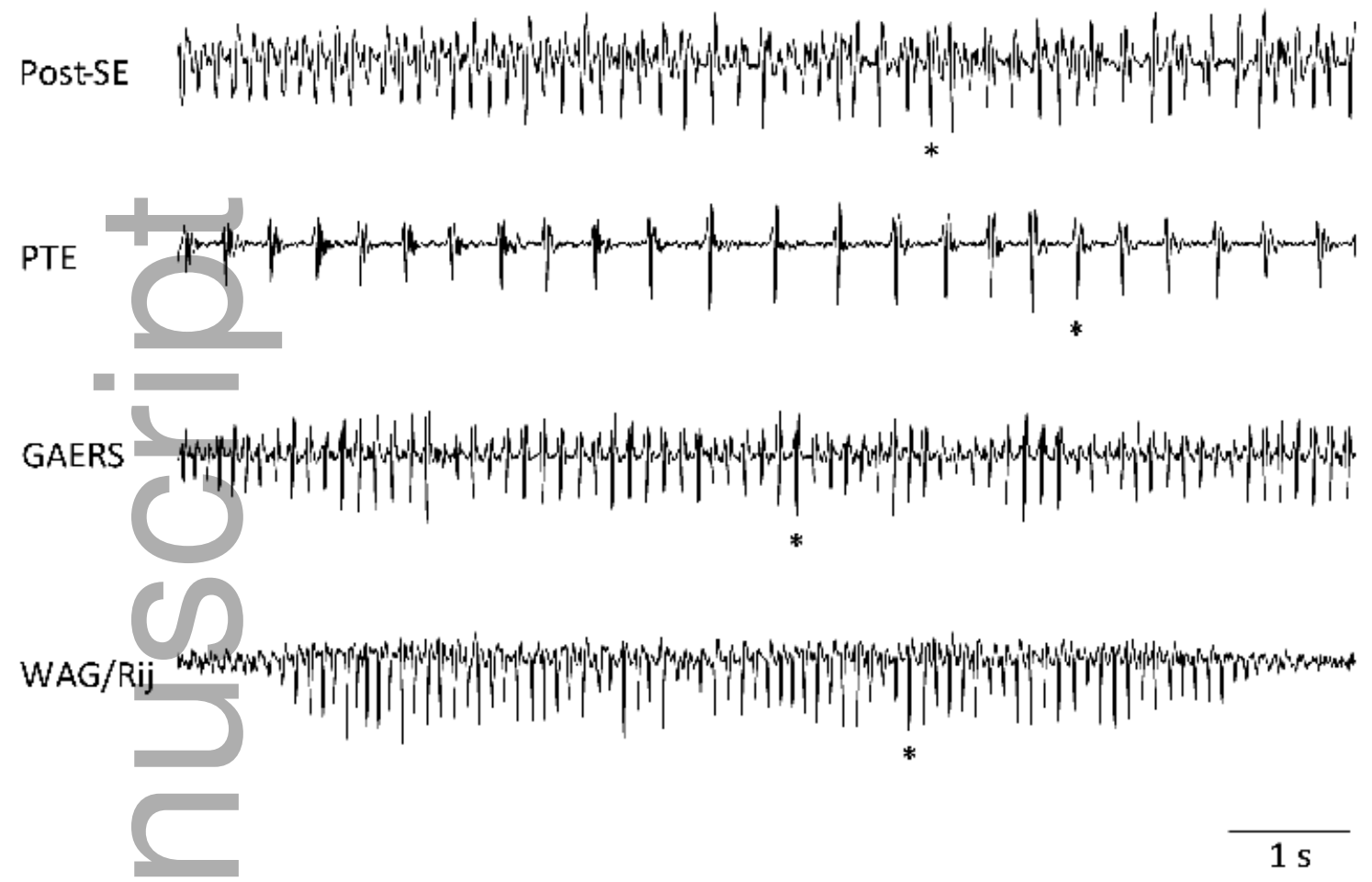

B


GAERS

WAG/Rij

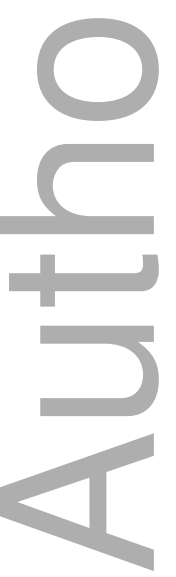

epi_14691_f3.tif

This article is protected by copyright. All rights reserved 


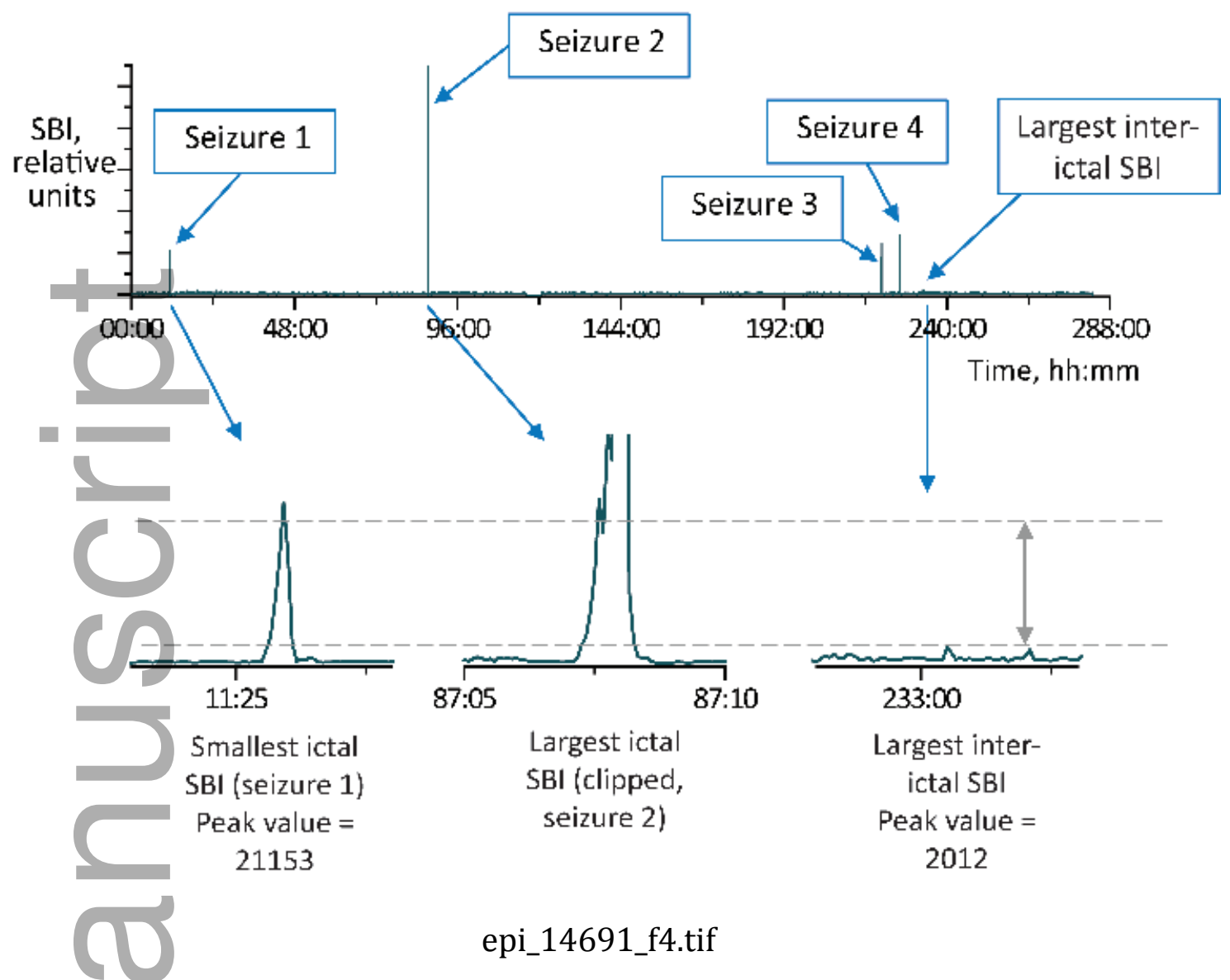

This article is protected by copyright. All rights reserved 


\section{University Library}

\section{- M M N E R VA A gateway to Melbourne's research publications}

Minerva Access is the Institutional Repository of The University of Melbourne

Author/s:

Casillas-Espinosa, PM;Sargsyan, A;Melkonian, D;O'Brien, TJ

Title:

A universal automated tool for reliable detection of seizures in rodent models of acquired and genetic epilepsy

Date:

2019-04-01

Citation:

Casillas-Espinosa, P. M., Sargsyan, A., Melkonian, D. \& O'Brien, T. J. (2019). A universal automated tool for reliable detection of seizures in rodent models of acquired and genetic epilepsy. EPILEPSIA, 60 (4), pp.783-791. https://doi.org/10.1111/epi.14691.

Persistent Link:

http://hdl.handle.net/11343/285576 